\title{
Error Estimation and Error Reduction in Separable Monte-Carlo Method
}

\author{
Bharani Ravishankar, $\_$Benjamin P. Smarslok, $₫$ Raphael T. Haftka, $₫$ and Bhavani V. Sankar $\stackrel{\S}{\$}$ \\ University of Florida, Gainesville, Florida 32611
}

DOI: $10.2514 / 1 . J 050439$

\begin{abstract}
Reliability-based design often uses the Monte-Carlo method as a sampling procedure for predicting failure. The combination of designing for very small failure probabilities $\left(\sim 10^{-8}-10^{-6}\right)$ and using computationally expensive finite element models, makes Monte-Carlo simulations very expensive. This paper uses an improved sampling procedure for calculating the probability of failure, called separable Monte-Carlo method. The separable MonteCarlo method can improve the accuracy of the traditional crude Monte-Carlo when response and capacity are independent. In previous research, accuracy of separable Monte-Carlo for a simple limit state was estimated via expectation calculus for a simple form of the limit state. In this paper, error estimates for a general limit state are developed through bootstrapping, and it is demonstrated that the estimates are reasonably accurate. Separable Monte-Carlo allows us to choose different sample sizes of the response and capacity in the limit state, and the paper demonstrates that bootstrapping may be used to estimate the contribution of the response and capacity to the total error. When the accuracy of the probability of failure is not good enough, the paper proposes reformulation of the limit state as another way to reduce uncertainty associated with the expensive random variable (usually the response). The accuracy of the bootstrapping estimates and the effectiveness of regrouping is demonstrated with an example of prediction of failure in a composite laminate with the Tsai-Wu failure criterion.
\end{abstract}

Nomenclature

$=$ in-plane stiffness matrix of the laminate

$=$ number of bootstrap samples

$b$

C

CV

$C V_{\text {boot }}$

$C V\left(\hat{p}_{\text {cmc }}\right)$

$C V\left(\hat{p}_{\text {smc }}\right)$

$C V\left(\hat{p}_{\mathrm{smc}}^{u}\right)$

$C V^{R}\left(\hat{p}_{\text {smc }}\right)$ system

$=$ coefficient of variation through bootstrapping

$=$ coefficient of variation of the crude Monte-Carlo technique

$=$ coefficient of variation of the original limit state

$=$ coefficient of variation of the regrouped limit state
$=$ coefficient of variation obtained probability of failure estimate from probability of failure estimate from separable Monte-Carlo method probability of failure estimate from separable Monte-Carlo method

$=$ uncertainty (coefficient of variation) in the probability of failure estimate due to response obtained empirically from separable Monte-Carlo method original limit state

Presented as Paper 2009-2266 at the 50th AIAA/ASME/ASCE/AHS/ASC Structures, Structural Dynamics, and Materials Conference, Palm Springs, CA, 4-7 May 2009; received 16 January 2010; revision received 19 May 2010; accepted for publication 10 June 2010. Copyright (C) 2010 by the American Institute of Aeronautics and Astronautics, Inc. All rights reserved. Copies of this paper may be made for personal or internal use, on condition that the copier pay the $\$ 10.00$ per-copy fee to the Copyright Clearance Center, Inc., 222 Rosewood Drive, Danvers, MA 01923; include the code 0001-1452/ 10 and $\$ 10.00$ in correspondence with the CCC.

*Graduate Student, Department of Mechanical and Aerospace Engineering; b2harani@ufl.edu (Corresponding Author).

Currently, Research Aerospace Engineer, Air Force Research Laboratory, Wright-Patterson Air Force Base, Ohio; smarslok@ufl.edu.

Distinguished Professor, Department of Mechanical and Aerospace Engineering; haftka@ufl.edu. Fellow AIAA.

${ }^{\S}$ Ebaugh Professor, Department of Mechanical and Aerospace Engineering; sankar@ufl.edu.

$$
\begin{aligned}
& C V^{C}\left(\hat{p}_{\mathrm{smc}}\right) \\
& \text { G } \\
& G^{u} \\
& \text { I } \\
& i, j \\
& \text { M } \\
& \operatorname{mean}\left(\hat{p}_{\mathrm{cmc}}\right) \\
& \operatorname{mean}\left(\hat{p}_{\text {smc }}\right) \\
& \operatorname{mean}\left(\hat{p}_{\text {smc,boot }}\right) \\
& \operatorname{mean}\left(p_{\mathrm{smc}}^{u}\right) \\
& \operatorname{mean}\left(p_{\mathrm{smc}, \mathrm{boot}}^{u}\right) \\
& \operatorname{mean}\left(\operatorname{stdev}^{R}\left(\hat{p}_{\text {smc,boot }}\right)\right)=\text { average uncertainty (standard } \\
& \text { deviation estimate) in the mean } \\
& \text { probability of failure estimate due to } \\
& \text { the response obtained by } \\
& \text { bootstrapping from separable } \\
& \text { Monte-Carlo method }
\end{aligned}
$$


mean $\left(\operatorname{stdev}^{C}\left(\hat{p}_{\text {smc,boot }}\right)\right)=$ average uncertainty (standard deviation estimate) in the mean probability of failure estimate due to the capacity obtained by bootstrapping from separable Monte-Carlo method mean $\left(\operatorname{stdev}\left(p_{\text {smc,boot }}^{u}\right)\right)=$ average of the standard deviation of the mean probability of failure estimate obtained by bootstrapping from separable Monte-Carlo method regrouped

$n$

$N$

P

$\bar{P}$

$p_{f}$

$\hat{p}_{\text {boot }}$

$\hat{p}_{\text {cmc }}$

$\hat{p}_{\text {smc }}$

$\hat{p}_{\mathrm{smc}}^{u}$

$[\bar{Q}]$

$R$

S

$\overline{\mathbf{S}}$

stdev $_{\text {boot }}$

$\operatorname{stdev}\left(\hat{p}_{\mathrm{cmc}}\right)$

$\operatorname{stdev}\left(\hat{p}_{\text {smc }}\right)$

$\operatorname{stdev}\left(\hat{p}_{\text {smc }}^{u}\right)$

$\operatorname{stdev}^{R}\left(\hat{p}_{\text {smc }}\right)$

$\operatorname{stdev}^{C}\left(\hat{p}_{\text {smc }}\right)$

$=$ uncertainty in the standard deviation of the probability of failure estimate due to the response obtained by bootstrapping from separable Monte-Carlo method original limit state

$\operatorname{stdev}\left(\operatorname{stdev}^{C}\left(\hat{p}_{\text {smc,boot }}\right)\right)=$ uncertainty in the standard deviation of the probability of failure estimate due to the capacity obtained by bootstrapping from separable Monte-Carlo method original limit state

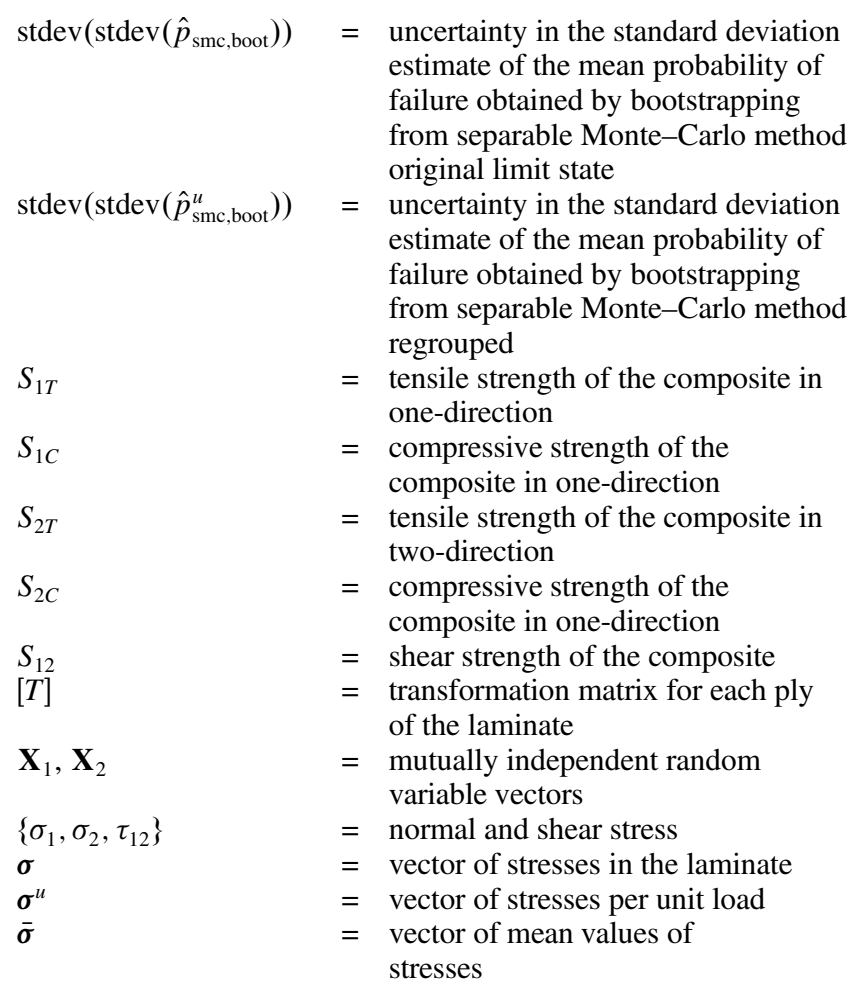

\section{Introduction}

A COMMON way to estimate the structural probability of failure $A$ of a system is with Monte-Carlo simulation of the capacity and response in the limit state function [1-6]. There are other more efficient methods, such as the first-order reliability method (FORM), second-order reliability method (SORM), but they do not work well for problems with multiple correlated failure modes $[\underline{7}, \underline{8}]$. The traditional, crude Monte-Carlo technique (CMC) is simple, but it lacks accuracy when sampling is limited due to computationally expensive structural analysis, such as from finite element analysis (FEA) [9]. When the response and capacity random variables are statistically independent, accuracy can be improved by the separable Monte-Carlo method (SMC) [10,11]. SMC samples response and capacity separately and compares all random samples of the response against all random samples of the capacity. This allows for improved accuracy of the calculation of the probability of failure for the same computational budget.

Previous work with separable Monte-Carlo only explored simple limit states, expressed as a difference between a random response and a random capacity [10]. For this simple limit state, error estimates were derived in terms of the number of samples of the response and capacity. This paper looks at a more general limit state function that combines sets of random response and capacity components. The first objective of the paper is to demonstrate that for the more general case, error estimates may be obtained by bootstrap techniques. A second objective is to demonstrate that these estimates may be used to guide uncertainty reallocation from expensive response to capacity for improved accuracy for a given computational budget.

Note that there are other techniques to improve the accuracy or efficiency of CMC, including importance sampling and the use of surrogates [12]. These complement SMC, in that they can be used together with SMC to further improve accuracy or efficiency.

The following section reviews the crude and separable MonteCarlo methods and illustrates the possible reallocation of uncertainty by calculating response per unit load. This section also describes the bootstrapping method, which is to be used for estimating the error in the SMC calculations. The crude and separable Monte-Carlo methods are then applied to the nonseparable limit state (the response and capacity components are integrated in the limit state) of the Tsai-Wu failure criterion for a composite pressure vessel in Sec. III. In Sec. IV, the accuracy of the methods is compared for different groupings of 
the random variables for the separable Monte-Carlo method from the estimate of coefficient of variation/standard deviation. The coefficient of variation of the probability of failure estimate for crude Monte-Carlo can be obtained from binomial law, whereas for separable Monte-Carlo, it is obtained from bootstrapping the limit state variables. The error in the standard deviation estimate of the bootstrapped probability of failure provides a measure of the accuracy of bootstrapping. Using SMC with the regrouped limit state reduces the error associated with the probability of failure estimate, since large samples of the inexpensive variables are available though the samples of expensive variables are limited. Further the expensive variables are bootstrapped and the accuracy of the bootstrapped probability of failure estimate is compared with that of the empirical estimate.

Since the example used in this paper involves simple calculations, the coefficient of variation estimate was also obtained empirically using different sample size of response and capacity. Comparing the bootstrapped estimate to the empirical estimate, would provide the magnitude of error associated with the bootstrapping technique.

\section{Probability of Failure Estimates}

Probability of failure is generally estimated using a limit state function $G$, which defines the failure condition. The limit state is a function of capacity $C$, and response $R$, which are assumed to be functions of statistically independent random variables $\mathbf{X}_{1}$ and $\mathbf{X}_{2}$, respectively. Equation (1) shows the separable case where failure occurs when a single component of response exceeds a single component of capacity

$$
G\left(\mathbf{X}_{1}, \mathbf{X}_{2}\right)=R\left(\mathbf{X}_{1}\right)-C\left(\mathbf{X}_{2}\right)
$$

Failure occurs when $G \geq 0$ and the system is safe when $G<0$. In the more general case, the capacity and the response in the limit state cannot be explicitly separated, and the limit state function may be represented as

$$
G\left(\mathbf{X}_{1}, \mathbf{X}_{2}\right)=G\left(R\left(\mathbf{X}_{1}\right), C\left(\mathbf{X}_{2}\right)\right)
$$

where $R$ and $C$ may be scalar or vector quantities.

\section{A. Crude Monte-Carlo Method}

A common simulation-based method for calculating the probability of failure $p_{f}$, is traditional, crude Monte-Carlo [1-5]. The probability of failure is estimated by comparing pairs of randomly generated response and capacity samples, as shown in Eq. (3)

$$
\hat{p}_{\mathrm{cmc}}=\frac{1}{N} \sum_{i=1}^{N} I\left[G\left(C_{i}, R_{i}\right) \geq 0\right]
$$

where $I$ is the indicator function, which equals one if the condition is true and zero if the condition is false. Thus, this essentially sums the number of failures for $N$ comparisons. The root mean square (rms) error in the estimate may be measured by the standard deviation for crude Monte-Carlo given as

$$
\begin{gathered}
\operatorname{stdev}\left(\hat{p}_{\mathrm{cmc}}\right)=\sqrt{\frac{p_{f}\left(1-p_{f}\right)}{N}} \\
C V\left(\hat{p}_{\mathrm{cmc}}\right)=\frac{\operatorname{stdev}\left(\hat{p}_{\mathrm{cmc}}\right)}{p_{f}}=\sqrt{\frac{\left(1-p_{f}\right)}{p_{f} N}} \approx \sqrt{\frac{1}{p_{f} N}}
\end{gathered}
$$

For example, for a probability of failure of 1 in.a million, 100 million simulations are needed for $10 \%$ error. The coefficient of variation calculated from standard deviation and mean provides a measure of the relative rms error in the probability of failure estimate about its mean value Eq. (5). Note that because we have only an estimate of the probability of failure, from Eq. (3) we get only an estimate of the error. The cost of response calculation is often the limiting factor in the number of samples $N$, because response calculations often require expensive finite element simulations.

\section{B. Separable Monte-Carlo Method}

When the capacity and response are statistically independent in the limit state, then they can be sampled separately using a method called separable Monte-Carlo. SMC has already been investigated for the simple limit state as shown in Eq. (1) [10]. This study looks at SMC for the general limit state in Eq. (2) Eq. (ㅁ)

$$
\hat{p}_{\mathrm{smc}}=\frac{1}{M} \frac{1}{N} \sum_{j=1}^{M} \sum_{i=1}^{N} I\left[G\left(C_{j}, R_{i}\right) \geq 0\right]
$$

where $N$ is the number of response samples and $M$ is the number of capacity samples. Because the response and capacity are sampled separately, all of the possible combinations can be considered to estimate $p_{f}$. Figure 1 illustrates the resulting difference between CMC and SMC.

In Fig. 1a, the direct one-to-one comparisons of CMC are shown for $N$ samples, whereas Fig. 1b shows that SMC looks at all of the possible combinations of random samples, which makes it inherently more accurate than CMC. In addition, different sample sizes can be used to enhance the accuracy, depending on the relative computational expense of the response and capacity.

\section{Error in the Probability of Failure Estimate}

For CMC, the rms error in the probability estimate is provided by Eq. (4). For SMC with the simple limit state as in Eq. (1), [10] derived analytical estimates of the standard deviation via expectation calculus. For the more general case of Eq. (2), we propose bootstrapping the components of the limit state $[\underline{1 \overline{3}}]$. For estimating the error in the probability of failure estimate, we use bootstrapping, a resampling technique, which involves taking the samples of response (expensive) and resampling them with replacement (so that the samples may contain duplicates). When we perform the resampling $b$ times, we obtain $b$ probability of failure estimates $\hat{p}_{\text {boot }}$. With $b$ estimates of $\hat{p}_{\text {boot }}$, we can obtain the standard deviation $\operatorname{stdev}\left(\hat{p}_{\text {boot }}\right)$ (see Fig. 2). As will be shown in the Sec. IV, the bootstrapping error estimates appears to be comparable to the CMC estimate of Eq. (4).

Furthermore, we can obtain $\hat{p}_{\text {boot }}$ estimates by bootstrapping capacity at mean values of response and vice versa. The knowledge of the individual contributions of the response and capacity towards the uncertainty aids in choosing the appropriate sample size for response $N$ and capacity $M$, which would provide an accurate estimate of the variation in the $p_{f}$ estimate. When we resample both the response and capacity, we obtain the total uncertaintystdev $\left(\hat{p}_{\text {smc,boot }}\right)$. But for the individual contributions of the response and capacity, the response has to bootstrapped at mean capacity and vice versa to obtain $\operatorname{stdev}^{R}\left(\hat{p}_{\text {smc,boot }}\right)$ and $\operatorname{stdev}^{C}\left(\hat{p}_{\text {smc,boot }}\right)$.

In the numerical results in the next section, the bootstrapping values are compared with the empirical values, $\operatorname{stdev}^{R}\left(\hat{p}_{\text {smc }}\right)$ and $\operatorname{stdev}^{C}\left(\hat{p}_{\text {smc }}\right)$ to demonstrate the accuracy of the bootstrapping

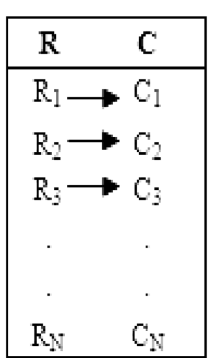

a)

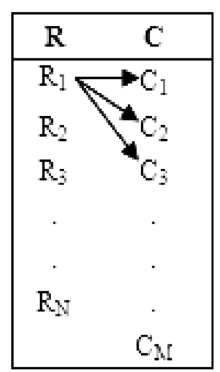

b)
Fig. 1 Illustration of crude and separable Monte-Carlo Method comparisons: a) CMC method, and b) SMC method where every sampled response is compared with every sampled capacity. 


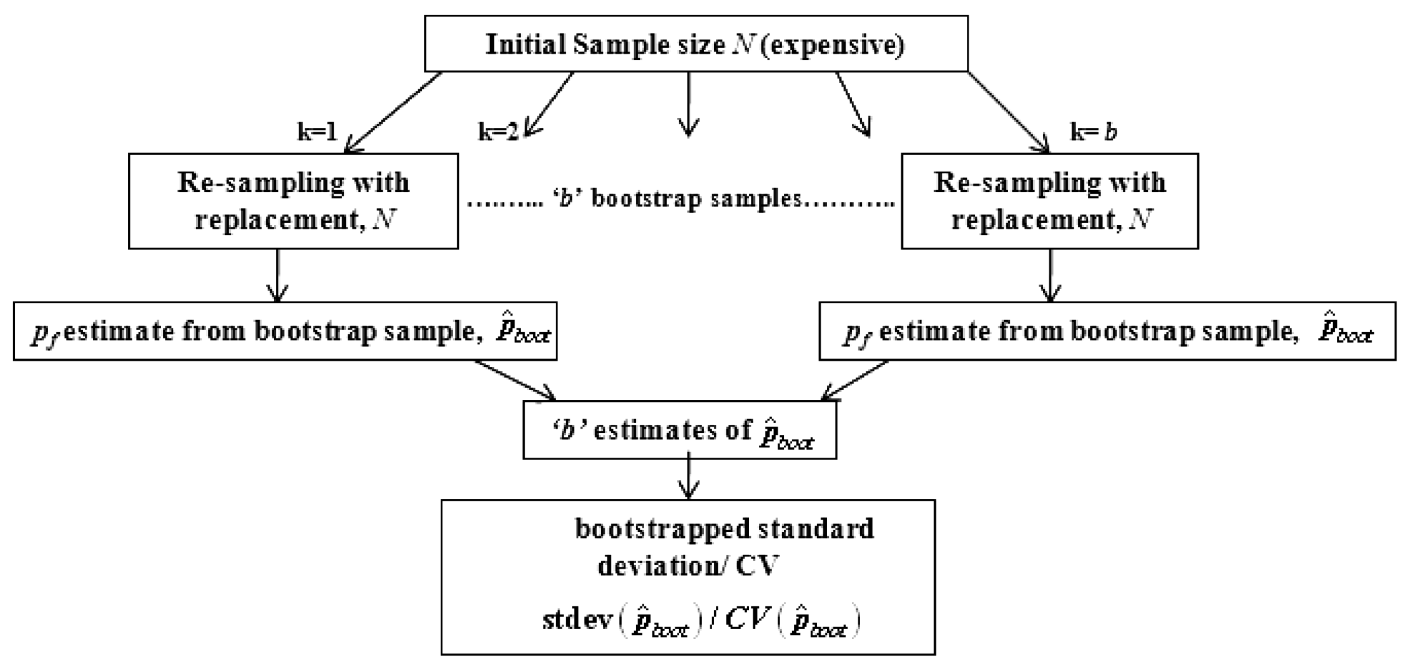

Fig. 2 Schematic representation of bootstrapping when only response is sampled.

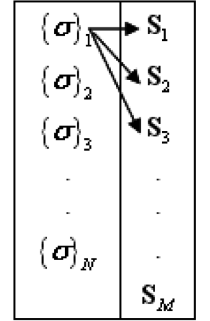

a)

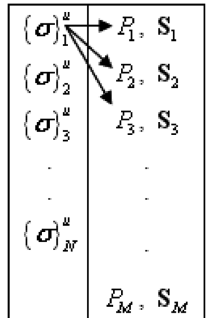

b)
Fig. 3 Illustration of separable sampling with unit loads: a) with stresses, and b) with unit load stresses.

method. To measure the error in the bootstrapped estimate, the uncertainty in the standard deviation $\left(\operatorname{stdev}\left(\operatorname{stdev}\left(\hat{p}_{\text {smc,boot }}\right)\right)\right)$ of the bootstrapped probability of failure estimate is also calculated. The bootstrapping estimates would allow us to judge whether the number of expensive simulations is sufficient for a desired level of accuracy.

\section{Separable Monte-Carlo with Regrouping and Separable} Sampling of the Limit State Random Variables

When the bootstrapping estimates show that the accuracy of the probability of failure is not good enough, and that the culprit is too few samples of the expensive simulation, we may have a painful choice between very high computational cost and accuracy. Often there is an alternative to increasing the number of expensive samples by reformulating the limit state, which is described in this section. The number of samples required for accurate modeling of the response and capacity depends on the relative contributions of the random components in the limit state function. Where the larger the uncertainty contribution, more samples are required for accurate
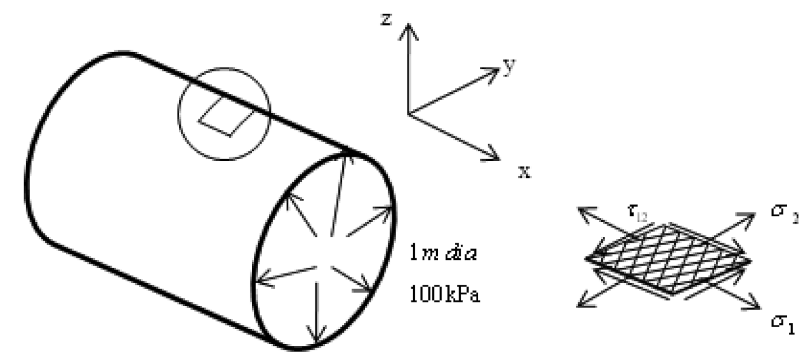

a)

b)

Fig. 4 Diagrams of: a) composite pressure vessel of $1 \mathrm{~m}$ diameter with an internal pressure of $100 \mathrm{kPa}$, and b) stresses acting in the laminate. representation of the distribution. Assuming a computational limit on the number of samples of the expensive response $N$, it is desirable to reduce the uncertainty in the response (i.e., obtain narrower distribution of the response) to achieve improved accuracy.

In most structural problems, failure of the system depends on the strength of the material $\mathbf{S}$ (e.g., capacity), and stresses $\sigma$ the structure sustains (e.g., response). So the limit state in Eq. (2) may become $G(\sigma, \mathbf{S})$. In linear problems, stresses $\sigma$, are a linear function of the load $P$, as in Eq. (7)

$$
\sigma=\sigma^{u} P
$$

where $\sigma^{u}$ are stresses per unit load. The randomness in the load $P$ is often independent of the random variables that affect $\sigma^{u}$ (geometry and material properties), but $P$ adds uncertainty to the computationally expensive stress calculation. Therefore, it would be advantageous to separate the loads from the stresses and determine stresses per unit load $\boldsymbol{\sigma}^{u}$. This also enables a larger sample size of the load. Even with a limited number of samples of stresses per unit load, the probability of failure can be more accurately estimated as both the strength and the load can be cheaply sampled. Then the expensive unit load response $\sigma^{u}$ is sampled and combined with the load in Eq. (7). Finally, the limit state is reformulated as

$$
G(\boldsymbol{\sigma}, \mathbf{S})=G^{u}\left(\boldsymbol{\sigma}^{u}, P, \mathbf{S}\right)
$$

The probability of failure can be determined from a large sample of loads and strengths compared with a limited sample of stresses, which is illustrated in Fig. 3.

The SMC formula that corresponds to Fig. 3 b is shown in Eq. (9). 1

$$
\hat{p}_{\mathrm{smc}}^{u}=\frac{1}{M} \frac{1}{N} \sum_{j=1}^{M} \sum_{i=1}^{N} I\left[G^{u}\left(\boldsymbol{\sigma}_{i}^{u}, P_{j}, \mathbf{S}_{j}\right) \geq 0\right]
$$

A similar form of Eq. (9) could be written for Fig. 3a, but with different indices.

\section{Application to Failure Analysis of Composite Laminate}

The CMC and separable sampling simulation methods and their error estimates are compared and illustrated by applying them to a nonseparable limit state problem [Eq. (2)]. References [14-16] discuss reliability-based optimization of composite laminates in detail. For complex structures, the stress is calculated through finite element analysis and it may be computationally expensive. To allow

IIt is possible to sample loads separately and with a different sample size than $M$. This would be an easy extension of SMC but for simplicity we do not consider it in this paper. 
Table 1 Material properties and uncertainty of the normally distributed random variables

\begin{tabular}{lccccc}
\hline \hline Properties & Mean & $C V \%$ & Strength & Mean & $C V \%$ \\
\hline$E_{1}, \mathrm{GPa}$ & 159.1 & $5 \%$ & $S_{1 T}, \mathrm{MPa}$ & 2312 & $10 \%$ \\
$E_{2}, \mathrm{GPa}$ & 8.3 & $5 \%$ & $S_{1 C}, \mathrm{MPa}$ & 1809 & $10 \%$ \\
$G_{12}, \mathrm{GPa}$ & 3.3 & $5 \%$ & $S_{2 T}, \mathrm{MPa}$ & 39.2 & $10 \%$ \\
$\nu_{12}$ & 0.253 & $5 \%$ & $S_{2 C}, \mathrm{MPa}$ & 97.2 & $10 \%$ \\
$P, \mathrm{kPa}$ & 100 & $15 \%$ & $S_{12}, \mathrm{MPa}$ & 33.2 & $10 \%$ \\
\hline \hline
\end{tabular}

us to perform thousands of Monte-Carlo simulations needed to validate the method, we selected an example that requires the calculation of stresses at a single point using Classical Lamination Theory (CLT).The problem involves prediction of failure of composite pressure vessel according to the Tsai-Wu failure criterion.

A composite laminate pressure vessel (Fig. 4) is made of a graphite/epoxy $[+25 /-25]_{s}$ symmetric laminate with each layer being $125 \mu \mathrm{m}$ thick and is subjected to an internal pressure of $100 \mathrm{kPa}$. The material properties of the composite are shown in Table 1. In this paper, all of the input random variables are assumed to be normally distributed. However, the performance of SMC depends on the distribution of the response and capacity, which is not necessarily normal. Previous research has used other distributions, such as lognormal and uniform, with SMC method [17,18].

The stresses generated are a function of the internal pressure $P$ and material properties of the laminate which are independent of each other.

$$
\begin{aligned}
& \left\{\begin{array}{c}
\sigma_{1} \\
\sigma_{2} \\
\tau_{12}
\end{array}\right\}=[T][\bar{Q}][A]^{-1}\left\{\begin{array}{c}
P d / 2 \\
P d / 4 \\
0
\end{array}\right\}=[T][\bar{Q}][A]^{-1}\left\{\begin{array}{c}
1 / 2 \\
1 / 4 \\
0
\end{array}\right\} P \\
& =\left\{\begin{array}{c}
\sigma_{1}^{u} \\
\sigma_{2}^{u} \\
\tau_{12}^{u}
\end{array}\right\} P
\end{aligned}
$$

The stresses $\sigma_{1}, \sigma_{2}$ (normal) and $\tau_{12}$ (shear) acting in each ply of the laminate are function of in-plane stiffness matrix of the laminate $[A]$, reduced stiffness matrix of each lamina $[\bar{Q}]$, transformation matrix of each lamina $[T]$, the pressure load $P$ and the diameter $d(1 \mathrm{~m})$ of the vessel as in Eq. (10). References [19-21] provide detailed explanation of Classical Laminate Theory $(\mathrm{CLT})$ and determination of stresses by CLT.

Failure of composite laminate is predicted from the strength of the composite and stresses generated using a suitable failure criterion. The most widely used criterion for composites is the Tsai-Wu criterion [19]. The criterion is a function of the strengths $\mathbf{S}$ (as shown

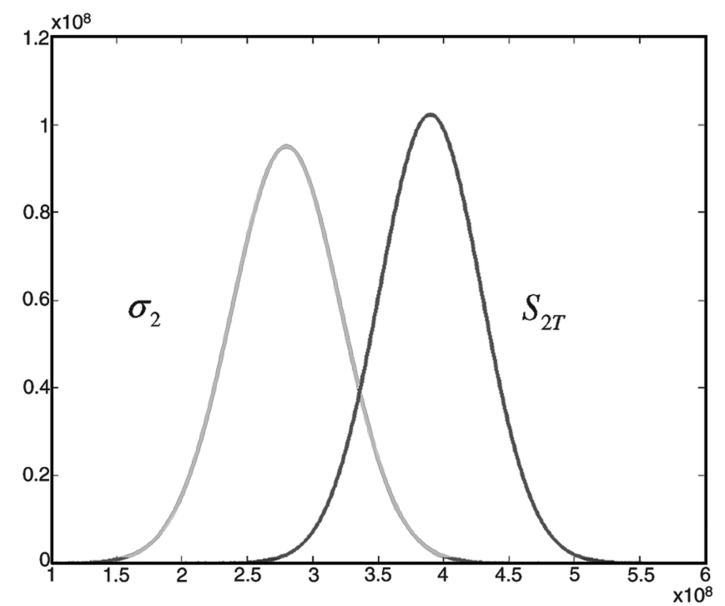

Fig. 5 Distribution of stress and strength in the two-direction $\sigma_{2}$ showing the probable failure region. in Table 1, [22] along with their uncertainties), and the stresses $\sigma$, in the fiber and transverse direction (e.g., one- and two-direction, respectively).

According to the criterion, a layer of the laminate is assumed to have failed when the limit state in Eq. (11) is greater than or equal to zero

$$
\begin{aligned}
& G(\mathbf{S}, \boldsymbol{\sigma})=F_{11} \sigma_{1}^{2}+F_{22} \sigma_{2}^{2}+F_{66} \tau_{12}^{2}+F_{1} \sigma_{1}+F_{2} \sigma_{2} \\
& \quad+F_{12} \sigma_{1} \sigma_{2}-1
\end{aligned}
$$

where

$$
\begin{array}{ccc}
F_{11} & =\frac{1}{S_{1 T} S_{1 C}} \quad F_{1}=\frac{1}{S_{1 T}}-\frac{1}{S_{1 C}} \quad F_{22}=\frac{1}{S_{2 T} S_{2 C}} \\
F_{2} & =\frac{1}{S_{2 T}}-\frac{1}{S_{2 C}} \quad F_{66}=\frac{1}{S_{12}^{2}} & F_{12}=\frac{\sqrt{F_{11} F_{22}}}{2}
\end{array}
$$

Uncertainties in the Tsai-Wu coefficients $\left(F_{11}, F_{22}, F_{66}\right.$, $\left.F_{1}, F_{2}, F_{12}\right)$ are due to randomness in the unidirectional tensile, compressive, and shear strengths $\mathbf{S}$ of the composite. Uncertainty in the stresses is due to randomness in material properties (or $\boldsymbol{\sigma}^{u}$ ) and pressure load $P$.

The in-plane normal and shear stresses $\left\{\sigma_{1}, \sigma_{2}, \tau_{12}\right\}^{T}$, are unique in each layer of the laminate. As previously mentioned, the laminate is made of $[+25 /-25]_{s}$ plies and the analysis shows that the inner $\left(-25^{\circ}\right)$ plies fail and then outer plies fail. Among the normal and shear stresses acting on the ply, the stress in the transverse direction $\sigma_{2}$ causes the failure of the laminate (the overlap of the stresses and strengths) which can be seen in Fig. $\underline{5}$.

The stresses are a function of material properties and internal pressure $P$ as in Eq. (10). For unit pressure load $(p=1)$, stresses are equal to $\boldsymbol{\sigma}^{u}$. Therefore, the original limit state function $(G(\sigma, \mathbf{S}))$ can be reorganized as indicated by Eqs. ( $\underline{6}-\underline{8})$.

\section{A. CMC and SMC Methods}

\section{Results and Discussion}

The probability of failure of a composite pressure vessel was calculated using CMC and SMC methods. It was assumed that our computational budget only permitted 500 stress calculations $(\sigma)$. Therefore, for $\mathrm{CMC}$, an equal number of random response $\sigma$ and capacity $\mathbf{S}$ variables $(N=500)$ were sampled for comparison. In the case of SMC, the response samples $(N)$ were compared against all the capacity samples $(M=500)$ resulting in 250,000 evaluations of the limit state. The actual probability of failure is $p_{f}=0.0121$ (As this is a simple problem, the actual probability of failure was estimated by CMC method by generating 5 million samples of stress and strength values). The relative error in crude Monte-Carlo was measured by calculating the standard deviation from Eq. (4) and hence the coefficient of variation. This value provides a measure of how accurate is the probability of failure estimate. For a simple limit state [as in Eq. (1)], the accuracy of separable Monte-Carlo can be estimated as derived by Smarslok et al. [10]. Because this problem is defined by a general limit state (e.g., Tsai-Wu), bootstrapping was performed to assess the accuracy of the probability of failure estimate of SMC with a $b=1,000$ bootstrap repetitions. Because the stresses are computationally expensive and it is cheaper to sample the strengths, random samples of stresses were bootstrapped, but the strengths were sampled anew rather than bootstrapped. That is, in each of the 1000 bootstrap repetitions, the stresses were resampled

Table 2 Empirical and bootstrapping estimates of probability of failure using SMC and CMC with $N=M=500$ and $n=10,000$ repetitions

\begin{tabular}{lcccc}
\hline \hline \multicolumn{1}{c}{ CMC } & \multicolumn{2}{c}{ SMC, original limit state } & \multicolumn{2}{c}{ SMC, regrouped } \\
\hline & Empirical & Bootstrapping & Empirical & Bootstrapping \\
\hline mean $\left(\hat{p}_{\text {cmc }}\right)$ & mean $\left(\hat{p}_{\text {smc }}\right)$ & mean $\left(\hat{p}_{\text {smc,boot }}\right)$ & mean $\left(\hat{p}_{\text {smc }}^{u}\right)$ & mean $\left(\hat{p}_{\text {smc,boot }}^{u}\right)$ \\
0.0121 & 0.0121 & 0.0121 & 0.0120 & 0.0122 \\
\hline \hline
\end{tabular}


Table 3 Standard deviation and coefficient of variation of empirical and bootstrapping $p_{f}$ estimates using SMC and CMC with $N=M=500$ and $n=10,000$ repetitions for original limit state. Standard deviation of the bootstrapping error is also shown

\begin{tabular}{lccccc}
\hline \hline \multicolumn{1}{c}{ CMC } & \multicolumn{2}{c}{ SMC, original limit state } & \multicolumn{2}{c}{ SMC, regrouped } \\
\hline \multicolumn{5}{c}{ Empirical } & \multicolumn{2}{c}{ Bootstrapping } \\
\hline $\operatorname{stdev}\left(\hat{p}_{\text {cmc }}\right)$ & $C V\left(\hat{p}_{\text {cmc }}\right)$ & $\operatorname{stdev}\left(\hat{p}_{\text {smc }}\right)$ & $C V\left(\hat{p}_{\text {smc }}\right)$ & $\operatorname{mean}\left(\operatorname{stdev}\left(\hat{p}_{\text {smc,boot }}^{u}\right)\right)$ & $\operatorname{stdev}\left(\operatorname{stdev}\left(\hat{p}_{\text {smc,boot }}^{u}\right)\right)$ \\
0.0048 & $40.0 \%$ & 0.0025 & $21.0 \%$ & 0.0020 & 0.0004 \\
\hline \hline
\end{tabular}

Table 4 Relative contributions of response (stresses) and capacity (strengths) toward the uncertainty in $p_{f}$ through bootstrapping and also compared with empirical results

\begin{tabular}{|c|c|c|c|}
\hline \multicolumn{2}{|c|}{ Empirical } & \multicolumn{2}{|l|}{ Bootstrapping } \\
\hline $\operatorname{stdev}^{R}\left(\hat{p}_{\mathrm{smc}}\right)$ & 0.0017 & $\operatorname{mean}\left(\operatorname{stdev}{ }^{R}\left(\hat{p}_{\text {smc,boot }}\right)\right)$ & 0.0019 \\
\hline$C V^{R}\left(\hat{p}_{\mathrm{smc}}\right)$ & $15.4 \%$ & $\operatorname{stdev}\left(\operatorname{stdev}^{R}\left(\hat{p}_{\text {smc,boot }}\right)\right)$ & 0.0004 \\
\hline $\operatorname{stdev}^{C}\left(\hat{p}_{\text {smc }}\right)$ & 0.0012 & $\operatorname{mean}\left(\operatorname{stdev}{ }^{C}\left(\hat{p}_{\text {smc,boot }}\right)\right)$ & 0.0014 \\
\hline$C V^{C}\left(\hat{p}_{\mathrm{smc}}\right)$ & $9.8 \%$ & $\operatorname{stdev}\left(\operatorname{stdev} C\left(\hat{p}_{\text {smc,boot }}\right)\right)$ & 0.0002 \\
\hline
\end{tabular}

from the same 500 sample, while the strengths had a fresh sample every time.

In this study (where simple CLT calculations were used) the accuracy of the separable Monte-Carlo method was also assessed by an empirical coefficient of variation obtained by performing $n=$ 10,000 repetitions. The probability of failure estimates are listed in Table 2 and the estimates of the error in the probability of failure are tabulated in Table 3. It shows that the coefficient of variation is reduced from 40 to $\overline{2} 1.0 \%$ by SMC. On average the bootstrapping error estimate of the SMC probability (as measured by the standard deviation) is about $20 \%$ low (0.002 compared with 0.0025$)$, with a standard deviation which is 5 times lower than that average. Thus in the large majority of cases the error estimate is within $50 \%$ of the empirical error.

Next we demonstrate obtaining the individual contribution of the response and capacity to the uncertainty in the probability of failure estimate obtained by bootstrapping the response at mean values of the capacity, and vice versa. The individual contributions would help when the overall error estimate is large and needs to be reduced by increased sample size. The values of mean $\left(\operatorname{stdev}^{R}\left(\hat{p}_{\text {smc,boot }}\right)\right)$ and mean $\left(\operatorname{stdev}^{C}\left(\hat{p}_{\text {smc,boot }}\right)\right)$ in Table $\underline{4}$ provide the uncertainty in $p_{f}$ estimate due to the stress and strength, respectively. These values are also compared with relative contributions of the stress and strength obtained empirically to illustrate the accuracy of the bootstrapping method.

From Table 4 we can see that the contribution of the response to the uncertainty in the $p_{f}$ estimate is higher than the contribution of the capacity. It is possible to reduce the response uncertainty by using a larger sample. However, since response calculation is usually expensive, we look instead to reduce the uncertainty in the response contribution by other means. The response contains the load with its large uncertainty $(C V(P)=15 \%)$. Calculating stress per unit load $\boldsymbol{\sigma}^{u}$, isolates the expensive CLT calculation (or FEA) from the large uncertainty in the load. The next section explores how the random components in the limit state can be reformulated by using unit stresses to reduce the error in the $p_{f}$ estimate.

\section{B. Regrouping and Separable Sampling of the Limit State Variables} for Improving Accuracy

In the original limit state $(G(\sigma, \mathbf{S}))$, the stress calculation contains the large uncertainty from the load. Therefore, rearranging the response to obtain stress per unit load $\sigma^{u}$, and load $P$ permits calculating response that does not include the uncertainty in the load. This arrangement will enable separable sampling of the independent variables of the limit state $G^{u}\left(\boldsymbol{\sigma}^{u}, P, \mathbf{S}\right)$, similar to that shown in Eq. (9). This regrouping shifts the large uncertainty in the load away from the expensive stress calculation $\left(\sigma^{u}\right)$. For $N=M=500 \quad(N=$ number of stress per unit load samples, $M=$ number of samples of strength and load), the uncertainty $\left(\operatorname{mean}\left(\operatorname{stdev}_{\text {boot }}^{R}\left(\hat{p}_{\text {smc }}\right)\right)\right)$ in the bootstrapped estimate for the reformulated limit state due to the response (unit load stresses) reduces to from 0.0019 to $8.6 \times 10^{-5}$. On the other hand, the capacity/ load uncertainty increases to from 0.0014 to 0.0044 . It would appear that we made the situation worse, but now we can reduce the error in $p_{f}$ estimate by increasing $M$, which is normally cheap. The value of $M$ was varied from 500 to 10,000 samples and the uncertainty in the estimate for reformulated limit state is shown in Table 5. It is clearly seen that the regrouping allows us to keep a small number of response calculations and reduce the uncertainty by having a very large number of inexpensive capacity (load) calculations. The standard deviation of the probability of failure for the regrouped limit state $\left(\operatorname{stdev}\left(\hat{p}_{\text {smc }}^{u}\right)\right)$ was also estimated empirically and shown in Table 5. The standard deviations obtained are plotted in Fig. 6.

Figure 6 clearly illustrates the effect of regrouping of the inexpensive random variables of the limit state. In the CMC method, the probability of failure is calculated using an equal number of response and capacity samples. In this case, 500 capacity samples and 500 response samples were used, which corresponds to a single value on the plot in Fig. $\underline{6}$.

In contrast, the SMC method can use different sample sizes $M$ for the random variables. Observe that the standard deviation from the original limit state of SMC levels off to a nearly constant value of 0.002 for $M$ samples greater than 5000. On the other hand, the standard deviation for the regrouped limit state continually decreases

Table 5 Standard deviation and coefficient of variation of CMC, SMC, and SMC-regrouped limit state for increasing sample size of $M$ and $N=500$. Bootstrapped and empirical estimates are shown

\begin{tabular}{|c|c|c|c|c|c|c|}
\hline \multirow[b]{2}{*}{$M$} & \multicolumn{2}{|c|}{$\mathrm{CMC}$} & \multicolumn{2}{|c|}{ SMC (empirical) } & \multicolumn{2}{|c|}{ SMC (bootstrapping) } \\
\hline & $\operatorname{stdev}\left(\hat{p}_{\mathrm{cmc}}\right)$ & $C V\left(\hat{p}_{\mathrm{cmc}}\right)$ & $\operatorname{stdev}\left(\hat{p}_{\text {smc }}\right)$ & $C V\left(\hat{p}_{\mathrm{smc}}\right)$ & $\operatorname{mean}\left(\operatorname{stdev}\left(\hat{p}_{\text {smc,boot }}\right)\right)$ & $\operatorname{stdev}\left(\operatorname{stdev}\left(\hat{p}_{\text {smc,boot }}\right)\right)$ \\
\hline 500 & 0.00488 & $40.0 \%$ & 0.0025 & $21.0 \%$ & 0.0020 & 0.0004 \\
\hline 5000 & & & 0.0021 & $17.6 \%$ & -a & - \\
\hline \multirow[t]{2}{*}{10,000} & \multirow{2}{*}{\multicolumn{2}{|c|}{$\mathrm{CMC}$}} & 0.0020 & $16.8 \%$ & - & - \\
\hline & & & SMC-regroup & (empirical) & \multicolumn{2}{|c|}{ SMC-regrouped (bootstrapping) } \\
\hline$M$ & $\operatorname{stdev}\left(\hat{p}_{\mathrm{cmc}}\right)$ & $C V\left(\hat{p}_{\mathrm{cmc}}\right)$ & $\operatorname{stdev}\left(\hat{p}_{\mathrm{smc}}^{u}\right)$ & $C V\left(\hat{p}_{\mathrm{smc}}^{u}\right)$ & $\operatorname{mean}\left(\operatorname{stdev}\left(\hat{p}_{\text {smc,boot }}^{u}\right)\right)$ & $\operatorname{stdev}\left(\operatorname{stdev}\left(\hat{p}_{\text {smc,boot }}^{u}\right)\right)$ \\
\hline 500 & 0.0048 & $40.0 \%$ & 0.0045 & $37.2 \%$ & 0.0046 & 0.0001 \\
\hline 5000 & & & 0.0015 & $12.6 \%$ & - & - \\
\hline 10,000 & & & 0.0009 & $7.9 \%$ & - & - \\
\hline
\end{tabular}

at was shown that bootstrapping provides uncertainty in the $p_{f}$ estimate with reasonably accuracy for $(N=M=500)$. It is possible to obtain the uncertainty in the $p_{f}$ estimate for increasing values of $M$, but it is not determined due to expensive computation. 


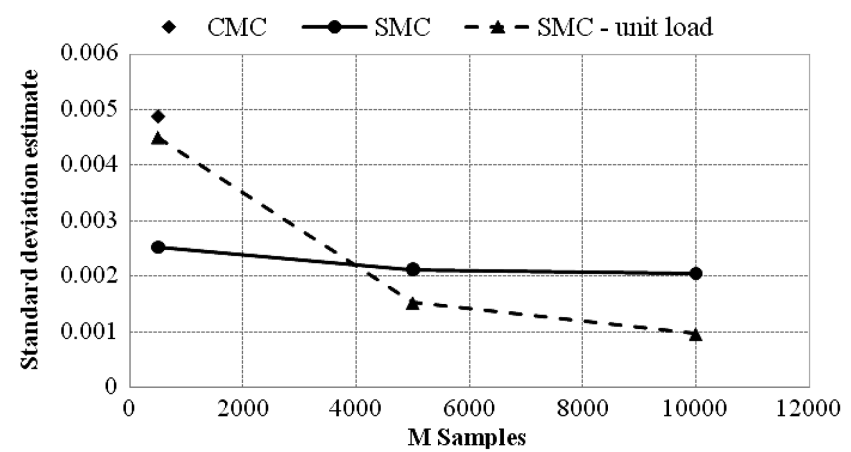

Fig. 6 Standard deviation of CMC, SMC, and regrouped limit state SMC where $N=\mathbf{5 0 0}$ (fixed) and $M$ is varying for 10,000 repetitions.

with the number of $M$ samples all the way to $\left(\operatorname{stdev}\left(\hat{p}_{\text {smc }}^{u}\right)\right) 0.0009$ (or $7.9 \% \mathrm{CV}$ ). In other words, in CMC the error estimate of the failure probability is $40 \%$, but the error associated in SMC is only $16.8 \%$ with the original limit state or $7.9 \%$ with the regrouped limit state. That is, for nearly the same computational cost, SMC with regrouping can estimate the failure probability more accurately than CMC.

Figure $\underline{6}$ shows that the magnitude of the uncertainty reduces with increase in number of $M$ samples. Thus by increasing the sample size of the inexpensive components (strength and the load), we could reduce the uncertainty in the $p_{f}$ estimate. For very large $M$, it would reach an asymptotic value due to the finite value of $N$. Figure 6 shows that by transferring some of the uncertainty from the response to the capacity, we can take advantage of increased $M$ to further reduce the error in the probability estimate.

\section{Conclusions}

The SMC method can provide substantial improvements in accuracy over the CMC method when response and capacity are governed by independent random variables. Obtaining estimates of the accuracy of SMC is critical to taking full advantage of the method. Here we proposed using bootstrapping to obtain estimates of the error in the SMC estimates, as well as the contributions of the capacity and the response to that error. The approach was demonstrated through an example problem of failure analysis of a composite pressure vessel using Tsai-Wu failure criterion. Because of the low computational cost of the example, it was possible to conduct multiple simulations and assess the accuracy of the bootstrapped estimate empirically.

SMC led to substantial accuracy improvement in determining the probability of failure compared with CMC method. Bootstrapping provided reasonable estimates of the uncertainty in the SMC probability of failure estimates. Bootstrapping also allowed estimating the individual contributions of the response and capacity toward the uncertainty in the probability of failure estimate, thus suggesting additional samples of inexpensive capacity would prove advantageous. Further substantial improvement in accuracy was achieved by transferring uncertainty away from expensive calculations by using unit load stresses and generating large samples of load and strengths.

\section{Acknowledgments}

This research is sponsored by NASA under a cooperative agreement (No. NNX08AB40A). Partial support was provided by a grant under the Constellation University Institutes Project. Any opinions, findings, and conclusions or recommendations expressed in this material are those of the author(s) and do not necessarily reflect the views of the National Aeronautics and Space Administration.

\section{References}

[1] Kalos, M. H., and Whitlock, P. A., Monte-Carlo Methods Volume I: Basics, Wiley, New York, 1986, pp. 92-103.

[2] Padmanabhan, D., Agarwal, H., Renaud, J. E., and Batill, S. M., "A Study Using Monte-Carlo Simulations for Failure Probability Calculation in Reliability-Based Optimization," Optimization and Engineering, Vol. 7, No. 3, 2006, pp. 297-316. doi:10.1007/s11081-006-9973-8

[3] Haldar, A., and Mahadevan, S., Probability, Reliability and Statistical Methods in Engineering Design, Wiley, New York, 2000.

[4] Rubinstein, R. Y., "Variance Reduction Techniques," Simulation and the Monte-Carlo Method, Wiley, New York, 1981, pp. 120-153.

[5] Qu, X., and Haftka, R. T., "Reliability-Based Design Optimization Using Probability Sufficiency Factor," Structural and Multidisciplinary Optimization, Vol. 27, No. 5, 2004, pp. 314-325.

[6] Ditlevsen, O., and Madsen, H. O., "Structural Reliability Methods," Wiley, New York, 1996.

[7] Di Sciuva, M., and Lomario, D., "A Comparison Between Monte-Carlo and Forms in Calculating the Reliability of a Composite Structure," Composite Structures, Vol. 59, No. 1, 2003, pp. 155-162. doi:10.1016/S0263-8223(02)00170-8

[8] Kim, N. H., Ramu, P., and Quiepo, N. V., "Tail Modeling in Reliability Based Design Optimization for Highly Safe Structural Systems," 47th AIAA/ASME/ASCE/AHS/ASC Structures, Structural Dynamics, and Materials Conference, AIAA, Reston, VA, May 2006.

[9] Tsompanakis, Y., and Papadrakakis, M., "Large-Scale ReliabilityBased Structural Optimization," Structural and Multidisciplinary Optimization, Vol. 26, No. 6, 2004, pp. 429-440.

[10] Smarslok, B. P., Haftka, R. T., and Kim, N. H., "Taking Advantage of Separable Limit States in Sampling Procedures," Proceedings of the 47th AIAA/ASME/ASCE/AHS/ASC Structures, Structural Dynamics, and Materials Conference, AIAA, Reston, VA, 2006.

[11] Smarslok, B. P., Alexander, D., and Haftka, R. T., "Separable MonteCarlo Simulation Applied to Laminated Composite Plates Reliability," 49th AIAA/ASME/ASCE/AHS/ASC Structures, Structural Dynamics, and Materials Conference, AIAA, Reston, VA, April 2008.

[12] Kalos, M. H., and Whitlock, P. A., "Monte-Carlo Evaluation of FiniteDimensional Integrals: Importance Sampling," Monte-Carlo Methods Volume I: Basics, Wiley, New York, 1986, pp. 92-103.

[13] Picheny, V., Kim, N. H., and Haftka, R. T., "Application of Bootstrap Method in Conservative Estimation of Reliability with Limited Samples," Structural and Multidisciplinary Optimization, Vol. 41, No. 2, 2009, pp. 205-217.

[14] Gurdal, Z., Haftka, R. T., and Hajela, P., Design and Optimization of Laminated Composite Materials, John Wiley and Sons, New York, 1999, Chap. 8.

[15] Qu, X., Venkataraman, S., Haftka, R. T., and Johnson, T. F., "Deterministic and Reliability-Based Optimization of Composite Laminates for Cryogenic Environments," AIAA Journal, Vol. 41, No. 10, 2003, pp. 2029-2036. doi: $10.2514 / 2.1893$

[16] Venkataraman, S., and Pablo, S., "Optimization of Composite Laminates for Robust and Predictable Progressive Failure Response," AIAA Journal, Vol. 45, No. 5, May 2007.

[17] Acar, E., Haftka, R. T., Kim, N. H., and Buchi, D., "Effects of Structural Tests on Aircraft Safety," Proceedings of the 50th AIAA/ASME/ASCE/ AHS/ASC Structures, Structural Dynamics and Materials Conference, AIAA Paper 2009-2265, Palm Springs, CA, 2009.

[18] Smarslok, B. P., "Measuring, Using, and Reducing Experimental and Computational Uncertainty in Reliability Analysis of Composite Laminates," Ph.D. Dissertation, Univ. of Florida, Gainesville, FL, 2009.

[19] Hyer, M. W., Stress Analysis of Fiber Reinforced Composite Materials, McGraw-Hill Science, New York, July 1997.

[20] Gibson, R. F., Principles of Composite Material Mechanics, McGrawHill, New York, 1994, pp. 190-269.

[21] Whitney, J. M., Structural Analysis of Laminated Anisotropic Plates, Technomic Publishing Company, Lancaster, PA, 1987.

[22] Stamblewski, C., Sankar, B. V., and Zenkert, D., "Three-Dimensional Quadratic Failure Criteria for Thick Composites using the Direct Micromechanics Method," 22nd Annual Technical Conference of the American Society for Composites, CD ROM Proceedings, Univ. of Washington, Seattle, WA, Sept. 2007.

A. Messac Associate Editor 